\title{
"Five on a Dice" Port Placement Allows for Successful Robot-Assisted Left Pneumonectomy
}

\author{
1 Division of Thoracic Surgery, Department of Surgery, \\ Houston Methodist Hospital, Houston, Texas, United States \\ Thorac Cardiovasc Surg Rep 2017;6:e42-e44.
}

Najah Khan ${ }^{1}$ Vid Fikfak ${ }^{1}$ Edward Y. Chan ${ }^{1} \quad$ Min P. Kim ${ }^{1}$

\begin{abstract}
Address for correspondence Min P. Kim, MD, Division of Thoracic Surgery, Department of Surgery, Houston Methodist Hospital, 6550 Fannin Street, SM1661, Houston, TX 77030-2707, United States (e-mail: mpkim@houstonmethodist.org).
\end{abstract}

\begin{abstract}
Background Technology has evolved to facilitate pulmonary resection. The latest technological advances in computer-aided surgery (Da Vinci Xi) allow for more control during pulmonary resection.

Keywords

- lung cancer treatment

- robotics

- minimally invasive surgery

Case Description A 59-year-old woman presented with two primary tumors of the left upper and lower lung. After induction chemotherapy, patient had a "five on a dice" port placement and technique was used to perform successful robot-assisted pneumonectomy. The patient was discharged home on postoperative day 3 without any complications. Conclusions We have found that the "five on a dice" port placement allows for optimal control of the robot stapler and facilitates successful robot-assisted left pneumonectomy.
\end{abstract}

\section{Introduction}

The surgical treatment of lung cancer patients has evolved over the years. Technological advancements have allowed surgeons to move from open thoracotomy to video-assisted thoracoscopic surgery (VATS) and now robot-assisted thoracoscopic surgery. The robot has evolved to provide better visualization and improved capabilities with each upgrade in technology. The latest version of the Da Vinci Xi provides better control during robot-assisted lung resection compared with the Da Vinci Si robot with the ability to control the vascular stapler. We have developed a port placement strategy that we term "five on a dice" allows us to perform robot-assisted pneumonectomy using robotic stapler.

\section{Case Description}

We utilized the "five on a dice" port placement and lung resection in the case of a 59-year-old former smoker, who originally presented with hemoptysis. A chest X-ray and a CT scan of the chest were significant for a $4.7 \mathrm{~cm}$ mass in left lower lobe (LLL). The lung lesion was then further evaluated by a PET-CT that showed uptake in the mass to 12.4 standard uptake value (SUVs) as well as a separate $1.4 \mathrm{~cm}$ left upper lobe mass with an uptake of $2.7 \mathrm{SUV}$. The patient underwent a biopsy of both lesions that was positive for adenocarcinoma in both sites. She then underwent induction chemotherapy with Alimta and cisplatin. After induction, she was restaged and found to have regression of the lower lobe tumor, while the upper lobe tumor remained stable in size. There was no lymph node involvement or metastatic disease. After a thorough workup and assessment of postoperative predictive lung function with pulmonary function testing, a ventilation-perfusion scan, brain MRI, and $\mathrm{mVO}_{2}$, the patient was recommended to undergo a robot-assisted pneumonectomy.

A 4-cm assistant port was placed in the fourth intercostal space in the mid-axillary line. A soft tissue retractor with a cap was used here to allow insufflation of the chest. The robotic camera port was placed in the seventh intercostal space just posterior to the posterior axillary line. An anterior $12 \mathrm{~mm}$ port was placed in the seventh intercostal space in the mid-axillary line and the posterior upper $8 \mathrm{~mm}$ port was placed in the seventh intercostal space by the scapula. Finally, the posterior lower $12 \mathrm{~mm}$ port was placed in the ninth intercostal space. This creates the "five on a dice" configuration (-Fig. 1). The robot is docked with the boom parallel to the seventh intercostal ports.

We placed the cadiere grasper in the left arm, the tip up instrument in the right upper port, and the bipolar dissector in the right lower port. We dissected the inferior pulmonary received

September 3, 2017 accepted after revision November 14, 2017
DOI https://doi.org/

10.1055/s-0037-1613714. ISSN 2194-7635. (c) 2017 Georg Thieme Verlag KG
Stuttgart · New York

License terms

$\Theta(1) \Theta \Theta$ 


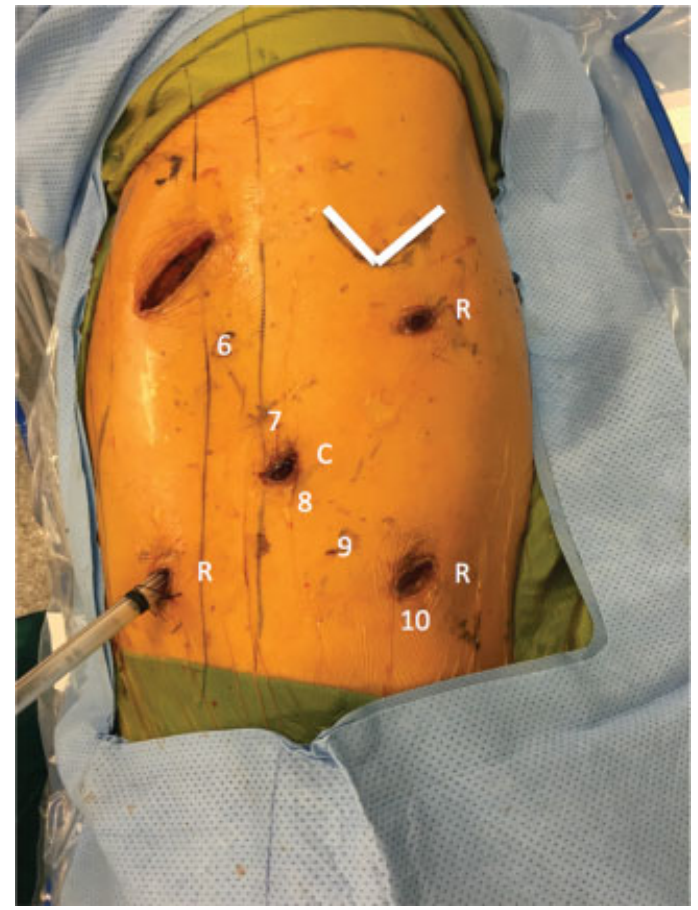

Fig. 1 Photograph of the "five on a dice" port placement for robotassisted left pneumonectomy. The assistant port is in the fourth intercostal space. Robot ports of $12 \mathrm{~mm}(\mathrm{R})$ are placed in the seventh intercostal space anteriorly and the ninth intercostal space posteriorly. A camera (C) is placed in the $8 \mathrm{~mm}$ robot port in the seventh intercostal space posterior to the posterior axillary line. The $8 \mathrm{~mm}$ robot port $(R)$ is placed by the tip of the scapula $(V /)$. ligament and the posterior hilum using the bipolar dissector. We then turned our attention to the anterior hilum and dissected out the inferior pulmonary vein, which we divided using a curved tip robotic vascular stapler from the anterior inferior $12 \mathrm{~mm}$ port (-Fig. 2A). Next, we dissected the superior pulmonary vein and divided it using the curved tip robotic vascular stapler from the posterior inferior $12 \mathrm{~mm}$ port (-Fig. 2B). Station 10L and 9 lymph nodes were harvested using the bipolar dissector. We proceeded by dissecting around the main bronchus, separating it away from the main pulmonary artery. Once the dissection was complete, we passed umbilical tape around the bronchus that allowed us to retract it away from the pulmonary artery. We then placed an EndoGIA tan load stapler through the anterior inferior port around the main pulmonary artery using an introducer and divided it (-Fig. 2C). Next, we performed flexible bronchoscopy to ensure that our staple line would be close to the main carina. We divided the bronchus using the robotic green stapler (-Fig. 2D) from the anterior inferior port. We then continued with dissection of station 5, 6, and 7 lymph nodes using the bipolar dissector. After ensuring no air leak was present at the bronchial stump, we placed a 32-French chest tube and closed the incisions in a layered fashion.

The patient tolerated the procedure well. She was transferred to the ICU for overnight observation and to the floor the following morning. The chest tube was removed on postoperative day 1 and she was discharged home on postoperative day 3 without any complications.

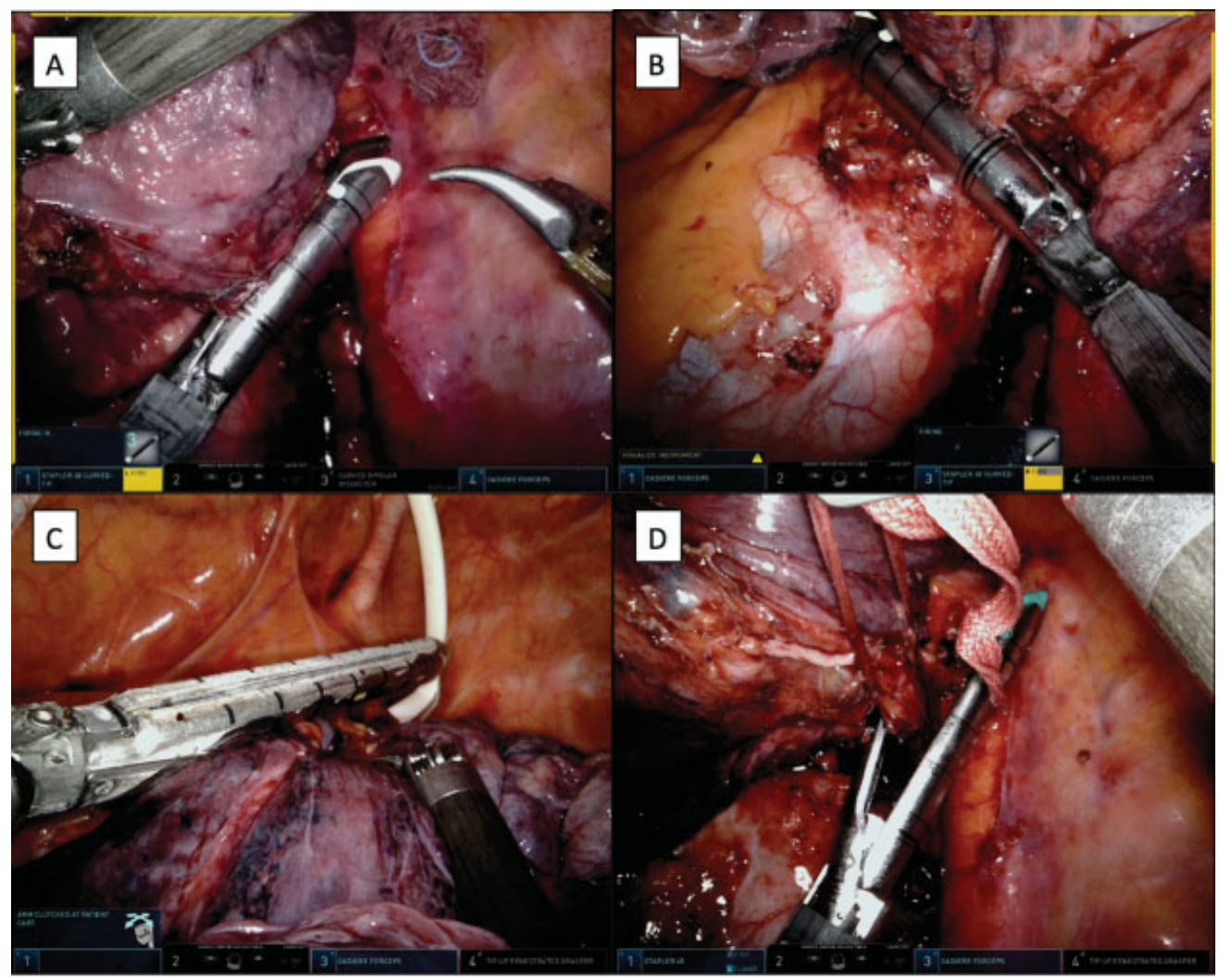

Fig. 2 Image of a stapled resection of hilar structures. The inferior pulmonary vein is divided with the robot stapler with the vascular load from the anterior inferior port (A); the superior pulmonary vein is divided with the robot stapler with the vascular load from the posterior inferior port (B); the main pulmonary artery is divided with the Endo-GIA tan load from the anterior inferior port (C); and the left main bronchus is divided with the robot stapler with a green load. 
e44 “Five on a Dice" Port Placement Khan et al.

\section{Discussion}

Several port placements have been developed to perform robot-assisted lung resection efficiently and ergonomically. Park has adopted the typical VATS port placement to use with the robot. ${ }^{1}$ Drs. Cerfolio ${ }^{2}$ and Dylewski ${ }^{3}$ developed the complete portal port placement that takes advantage of the Da Vinci Si robot's capabilities. Both of these strategies were developed for the Da Vinci S/Si platform where there was no vascular stapler that could be controlled by the surgeon. With advent of the Da Vinci Xi and its ability to control the vascular stapler, the "five on a dice" configuration of the ports allows us to insert and use the robotic stapler via either the left or the right inferior ports and provide improved control of the vascular staplers during the case. This port placement and technique allow for the successful completion of challenging cases such as pneumonectomy. Further improvements with the Xi system are necessary to make this case less dependent on an assistant. If there were a $60 \mathrm{~mm}$ robot vascular stapler, the division of the main pulmonary artery could have been accomplished by the surgeon sitting on the robot console. Robotic pneumonectomy is a challenging operation, but the use of the "five on a dice" port placement and technique takes full advantage of the Da Vinci Xi's capabilities and allows for safe and ergonomic lung resection.

\section{Acknowledgments}

No financial support or grant was received for this work. MPK and EYC have a consulting agreement with Boston Scientific, Medtronics, and Olympus.

\section{References}

1 Park BJ. A complete video-atlas of five robotic-assisted lobectomies. Ann Cardiothorac Surg 2012;1(01):100-101

2 Cerfolio RJ, Bryant AS. Robotic-assisted pulmonary resection - right upper lobectomy. Ann Cardiothorac Surg 2012;1(01):77-85

3 Ninan M, Dylewski MR. Total port-access robot-assisted pulmonary lobectomy without utility thoracotomy. Eur J Cardiothorac Surg 2010;38(02):231-232 